\title{
Sports Activities in Obese Teenagers Improve Social Inclusion and Health
}

\author{
Pietro Montesano ${ }^{1}$ and Filomena Mazzeo ${ }^{2}$ \\ 'University of Naples Parthenope, Department of Motor Sciences and Wellness, Naples, Italy, ${ }^{2}$ University of Naples Parthenope, Department of \\ Science and Technology, Naples, Italy
}

\begin{abstract}
Obesity is known to be a major risk of whole range of cardio vascular, metabolic and respiratory disorders. The etiology of obesity can be genetic, hormonal and determine the central obesity (or visceral or android). It is characterized by the deposit of fat especially abdominal and recognizable by a waist-hip ratio of more than 0.85 in women and 0.95 in 'man, and the peripheral (or subcutaneous or gynoid) that shows a ratio of less than 0.85 in women and 0.95 in men. The research was carried out in order to monitor and improve the correct use of the respiratory function of obese adolescents by developing a sports program aimed at reducing body weight and enhancing motor skills on 60 subjects (30 males and 30 females) aged between 14 and 16 years for a period of eight months. Results showed a reduction in body weight for almost all participants. For 26 subjects, the reduction was around $10 \%$, while for others it was around $8 \%$. Only 8 subjects did not significantly change their weight. The improvement of the motor program, on average, was about $9.5 \%$. The phenomenon of obesity is often one of the factors of discomfort conditions in adolescence that slows down the process of social inclusion and highlights "paramorhism" that has negative effect on the respiratory function. Sport is one of the most important activities to combat the problem of weight gain because, through the rules of sports disciplines, it favors the acquisition of correct lifestyles.
\end{abstract}

Key words: obesity, sport, exercise testing, pulmonary function tests, inclusion

\section{Introduction}

Obesity is known to be one of the most important health problems in the U.S and in other countries (Oates et al., 2018; Mazzeo, 2016). The World Health Organization (WHO, 2013) predicts that, by 2015 , around 700 million adults will be obese (at least $10 \%$ of the projected global population). Also, obesity is known to be a major risk of whole range of cardio vascular, metabolic and respiratory disorders (Mazzeo, 2016; Boulet, 2013.) The obese adolescent presents, in addition to the characteristics of an organic nature, mainly those of a psychological nature. The body transformation determined by age and the often negative judgments of peers frequently determine psychological problems with personality disorders and with attempts, often inappropriate, of compensation on the body of affective-relational disorders (Illiano et al., 2017). Furthermore, obesity it is often one of the factors of the conditions of discomfort in the adolescent age that slows down the process of social inclusion and it degrades the functionality of large body systems (Salome, King, \& Berend, 2010). The problematic of increasing body weight is the result of a glucidic and protidic energy imbalance (Hill et al., 2018; Wells, Noseworthy, Hamilton, Tarnopolski, \& Tein, 2008) prolonged over time; in practice, children, adolescents, but also adults, introduce a surplus of calories than the body actually needs. The global approach to obesity (Mazzeo, 2016) is often difficult, caused by the multifactorial nature of the phenomenon. In the etiopathogenesis there are multiple factors, both environmental and psychological (Zametkin, 2004), both of a genetic nature (Illiano et al., 2017). The treatment of obesity cannot and should not be understood as a simple prescription of certain diets or a simple encouragement to perform motor-sports activities (Yazdani, Sharif, Elahi, Ebadi, \& Hosseini, 2018).

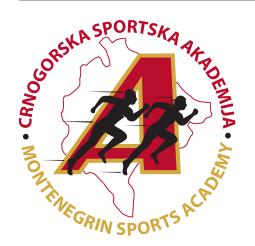

Correspondence: 
In the scholastic and social context, the phenomenon has assumed increasingly significant and worrying connotations since for adolescents the physical, aesthetic, and the difficulty in actively participating in a life of gratifying relationship gradually determines conditions of discomfort. In some cases of marginalization with psychosomatic repercussions that reflect on the correct use of large systems (P.B. Persson, Bondke, \& A. Persson, 2018; Schenone et al., 2003). Obesity has been commonly associated with an increased risk of developing new cases of asthma. Also, it has been difficult to define the ways through which obesity affects the asthma phenotype in children and adolescents (Farah \& Salome, 2012). Epidemiological data showed that obesity has various effects on respiratory system and seems to be a predisposing factor for the development of asthma and leads the development of asthma raises (Perrotta, Mazzeo, \& Cerqua, 2017). Obese patients with asthma more symptoms and increased morbidity compared with non-obese asthma patients (Boulet, 2013; Matera, Rinaldi, Calzetta, \& Cazzola, 2017). The major respiratory complications of obesity contain a heightened demand for ventilation, elevated work of breathing, respiratory muscle inefficiency and diminished respiratory compliance (Parameswaran, Todd, \& Soth, 2016).

Furthermore, study showed that he physical activity such as aerobic or anaerobic exercise alters the respiratory parameters on obese population. Obese can intensely alter pulmonary function and diminish exercise capacity by its adverse effects on respiratory mechanics, resistance with in the pulmonary system, respiratory muscle function, lung volumes, work and energy cost of breathing, and gaseous exchange (Luce, 1980; Mazzeo 2016). In the population, the widespread tendency of adolescents to be overweight or obese, and this number is continuing to rise, exposed the Children have fewer weight-related health and medical problems than adults. However, overweight children are at high risk of becoming overweight adolescents and adults, placing them at risk of developing chronic diseases such as heart disease and diabetes later in life. They are also more prone to develop stress, sadness, and low self-esteem (Mazzeo, 2016). In obese people the benefits of various forms of physical activity and response with long term follow up need to be further assessed (Salome et al., 2010).

The diagnosis of obesity, in addition to the irrefutable objective examination and body weight, is carried out in relation to the concept that the human body consists of two compartments:

- fat mass (FM=fat mass): consisting of all body lipids distributed in the subcutaneous and visceral tissues;

- lean mass (FFM=free fat mass): consisting of muscle mass, bone and inter and intra-parenchymal non-adipose tissues.

The concept of fat mass and lean mass is substantiated in the identification of specific indexes of reference, BMI or IMC, which are calculated through pre-established formulas, $\mathrm{BMI}=$ weight $(\mathrm{kg}) /$ stature $(\mathrm{m} 2)$, whose results provide ranges (Tables 1 and 2) which determine the categories at risk.

Table 1. Reference range of the values of the IMC

\begin{tabular}{ccc}
\hline INDEX & VALUE & CONDITION \\
\hline IMC & $<18$ & situation of underweight \\
IMC & $18.5-25$ & optimal weight situation \\
IMC & $25.1-30$ & overweight situation \\
IMC & $30.1-40$ & obesity situation \\
IMC & $>40$ & situation of severe obesity \\
\hline
\end{tabular}

Table 2. Reference range of the values of the BMI

\begin{tabular}{ccc}
\hline INDEX & VALUE & CONDITION \\
\hline BMI & 24.9 & upper limit of normality \\
BMI & $25-29.9$ & obesity of the first degree \\
BMI & $30-39.9$ & obesity of the second degree \\
BMI & $>40$ & WWobesity of the third degree \\
IMC & $>40$ & situation of severe obesity \\
\hline
\end{tabular}

To investigate the effect of obesity on respiratory system, most researches used values of pulmonary function test (PFT). The spirometry tests measured were the forced vital capacity (FVC), forced expiratory volume in one second (FEV1), peak expiratory flow rate (PEFR) and forced mid-expiratory flow (FEF 25-75\%) and ratio of FEV1 to FVC was calculated to find the impact of obesity on ventilation. But most of the studies focus on FEV1, minute ventilator volume and ERV. Obese children have more respiratory symptom than their normal weight peers.

\section{Method}

The study carried out, with an observational method, on a sample of 60 subjects, 30 men and 30 women, aged between 14 and 16 years with a body weight of between $65 \mathrm{~kg}$ and 80 $\mathrm{kg}$. The specific objective of the research, was to verify the im- provement of the inclusion and the reduction of body weight, thought the execution of two hours of extra weekly extracurricular training. The activity performed in an indoor plant taking care the strengthening of the coordinative abilities, in particular of the oculo-manual and oculo-podalica coordination, of the spatio-temporal organization and of the rhythm as well as of the ability to work in groups (Comoglio \& Cardoso, 1996) through the use of team sports disciplines. All 60 subjects participated frequently in additional training sessions.

At the beginning and at the end of the sports route, motor tests were administered (Marella \& Risaliti, 2007) to monitor not only the reduction of body weight, but also progress on the use of coordination skills (Magni, 2009) and conditional (Montesano et al., 2013) and the parameters of respiratory function as spirometry. 


\section{Additional training}

The sporting path was developed with four training sessions weekly, each carried out on different days, two of which in the morning hours and two in the afternoon. During the first fifteen days interviews centered on the knowledge of correct eating habits and on the importance of sporting activity in adolescence were carried out and only the anti-daily sessions were held (Montesano, 2018).

In the following months, all four sessions were carried out, increasing the duration in the afternoon sessions (average from $30-45 \mathrm{~min}$ to $55-70 \mathrm{~min}$ ) and the intensity of the activity. In a standard work session the intensity should be low at least for the first fifteen minutes, with a steady and steady heart rate and a continuous and regular oxygenation. In fact, aerobic training, in addition to the direct consumption of fats, induces positive changes in the basal metabolism; greater resistance in the activities of daily life; greater resilience after every type of effort; a greater supply of blood to the brain and muscles; a regularization of arterial pressure; a regularization of the pulse frequency. The coaching sequence was carried out with 10 'of slow running, 8' of mobilization exercises, 3 'of respiratory gym exercises, 12' exercises with the use of small tools and with small weights, 6 'of abdominal exercises and backbones, 10 'ball games, 3 ' of stretching exercises, 8 'of slow running (Montesano, 2016).

The exercises were set up with the concept of work in progress organizing work paths alternating coordinative and conditional exercises. The organization of work in circuits has provided for the enhancement of oculo-manual and oculo-podalica coordination, organization of space, rhyt$\mathrm{hm}$, the specific technique of the most common team games such as basketball, football (Montesano, 2016), volleyball, handball. The exercises were initially performed at natural load using balloons, cones, rods, carpets, elastics, circles. Only from the fifth month were introduced medicinal flasks, ballasted anklets and other small tools. There were also sessions dedicated to free play. These sessions were preparatory to those that, developed during team games, stimulated the issues of collaboration, the development of unforeseen gaming situations, with the related strategies to be adopted for the resolution of the problems present during a race also through breathing control.

Materials and resources: 1) Indoor Stadium; 2) Small tools (Clavette, supports, circles, sticks, elastics); 3) Basketball and soccer ball; 4) 1,2,3,5 kg medicinal ball; 5) 1.2 and $3 \mathrm{~kg}$ weights; 6) Ballasted anklets of 1,2,3, kg; 7) Detection grids; 8) Spirometer

\section{Results}

The results were elaborated by measuring, at the beginning and at the end of the research path, the weight of the participants, highlighting the initial and final average weight measured after eight months of activity.

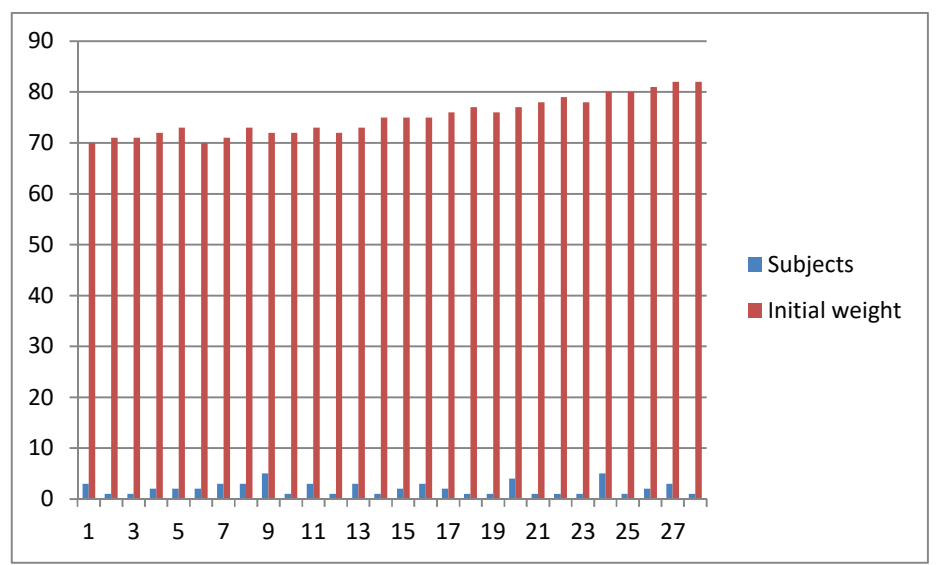

Figure 1. Initial detection average weight 60 subjects

The average reduction in body weight was around $9 \%$. Twenty-six subjects decreased their weight by about $10 \%$ while sixteen subjects decreased it by $8 \%$. Only eight subje- cts did not register significant weight variations. Research has shown a reduction in $\mathrm{kg}$ (Figure 1 and 2).

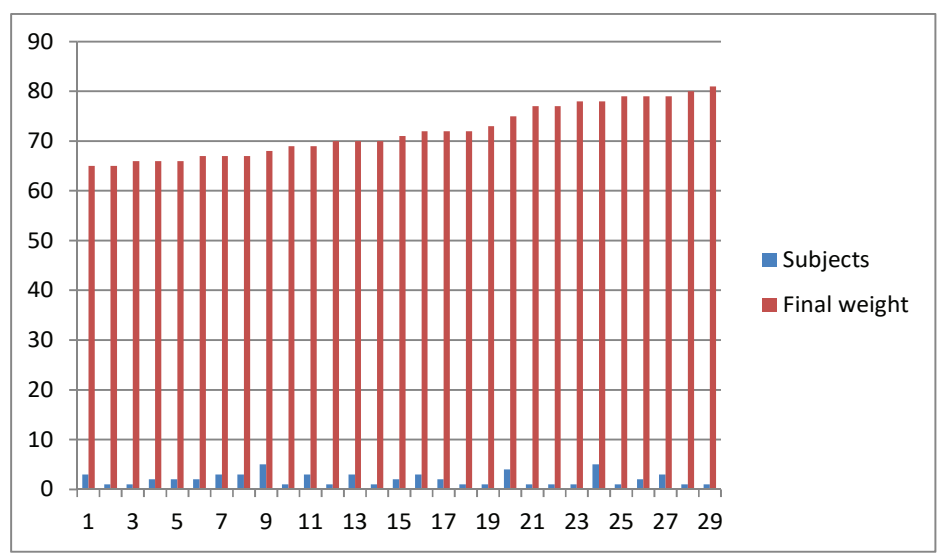

Figure 2. Final detection average weight 60 subjects 
This is more favorable for men than for women. The comparison between initial and final weight showed (Table 3 , Figure 3 ) that only three subjects did not benefit from we- ight reduction while the other fifty-seven showed reductions in body weight.

Table 3. Comparison between initial and final weight

\begin{tabular}{|c|c|c|}
\hline $\begin{array}{l}\text { Number of } \\
\text { Subjects }\end{array}$ & Initial weight & Final weight \\
\hline 3 & 70 & 65 \\
\hline 1 & 71 & 65 \\
\hline 1 & 71 & 66 \\
\hline 2 & 72 & 66 \\
\hline 2 & 73 & 66 \\
\hline 2 & 70 & 67 \\
\hline 3 & 71 & 67 \\
\hline 3 & 73 & 67 \\
\hline 5 & 72 & 68 \\
\hline 1 & 72 & 69 \\
\hline 3 & 73 & 69 \\
\hline 1 & 72 & 70 \\
\hline 3 & 73 & 70 \\
\hline 1 & 75 & 70 \\
\hline 2 & 75 & 71 \\
\hline 3 & 75 & 72 \\
\hline 2 & 76 & 72 \\
\hline 1 & 77 & 72 \\
\hline 1 & 76 & 73 \\
\hline 4 & 77 & 75 \\
\hline 1 & 78 & 77 \\
\hline 1 & 79 & 77 \\
\hline 1 & 78 & 78 \\
\hline 5 & 80 & 78 \\
\hline 1 & 80 & 79 \\
\hline 2 & 81 & 79 \\
\hline 3 & 82 & 79 \\
\hline 1 & 82 & 80 \\
\hline 1 & 81 & 81 \\
\hline
\end{tabular}

At the end of the course, the participants were interviewed again and everyone declared that the experience produced positive effects both from an aesthetic and functi- onal point of view, and from the aspect of breathing control that reduces the manifestations of anxiety.

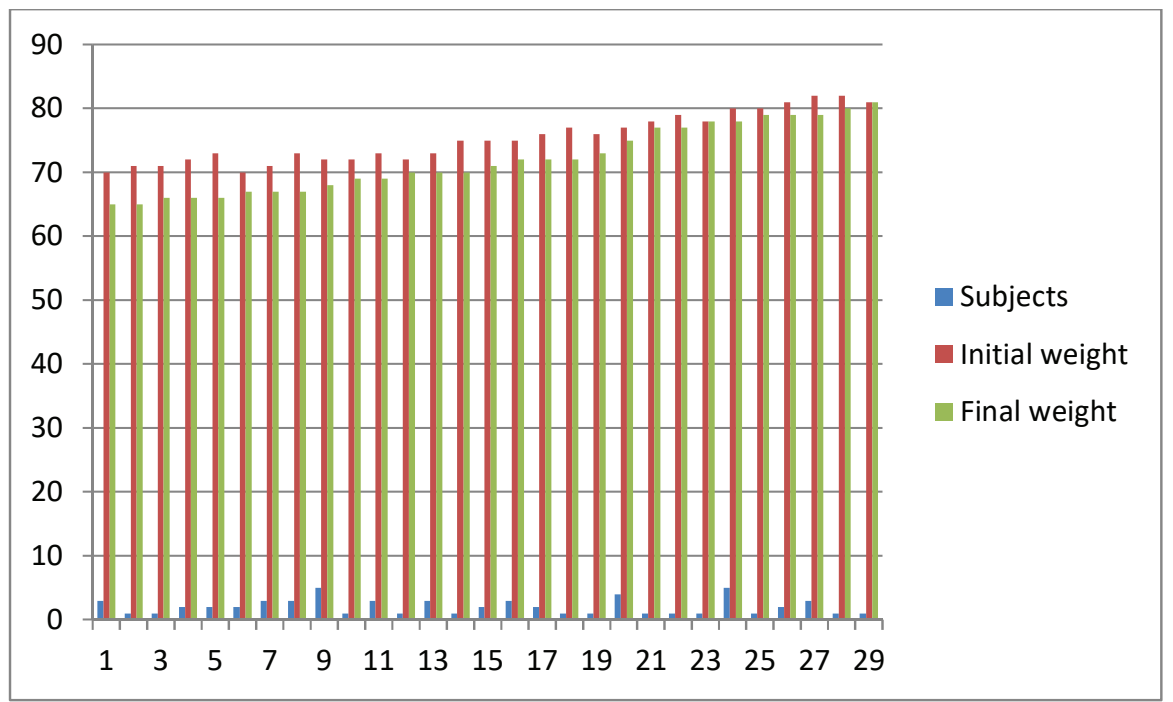

Figure 3. Comparison between initial and final weight 


\section{Discussion}

The drafting of a suitable sporting work path is based on the knowledge of the condition of subjects at risk of obesity (the daily human lipid energetic requirement is between 0.8 $1.2 \mathrm{gr} / \mathrm{kg}$ of body weight while the glucide is between $100-150$ $\mathrm{gr} / \mathrm{kg}$ of body weight). Furthermore, on the awareness that the physical-sport activity, carried out in a continuous and regular way, induces benefits effects on weight control, on the regulation of energy expenditure, on cardio-circulatory and respiratory function (Mazzeo, 2016). Most of the studies showed that there was a direct improvement on respiratory parameters in obese people either with aerobic or anaerobic exercise (Vengata, Ganesh, \& Wan Rosley, 2014). Benefit showed also on the functionality of the osteoarticular and muscular structures, on the capacity for coordination and dexterity, on the structuring of character, self-control and self-esteem (Ruiz et al., 2010) (Table 3). A healthier body means feeling good about yourself. The fragmentation and the lack of synergy of the interventions, such as the prescription of a diet or the generic invitation to the performance of a not well identified physical activity, do not meet the appropriate requirements for the treatment of obesity. The multidisciplinary approach, oriented to monitoring pathology and ongoing support, represents the appropriate path to be pursued to obtain effective results (Montesano \& Mazzeo, 2018).

At a general level, the physical activity performed regularly and lasting at least thirty minutes a day, combined with an adequate diet, determines, in a medium-long period, an increase in energy expenditure and therefore a corresponding decrease in fat (Mazzeo, 2016; Mazzeo et al., 2018). In the scholastic context, a condition in which learners spend long hours seated, it is necessary to balance this indication with curricular and extra-curricular activities that favor aerobic and low intensity activities but, above all, group activities that foster peer relationships. The condition of adolescent obesity is a widespread problem within the social and educational community. The dynamics of the developmental age are amplified if the adolescent is affected by obesity and the good motor practices can be the key to obtaining an effective inclusive result. It is therefore necessary that health be protected through movement and to the benefits that physical activity induces both to maintain an adequate psychophysical balance and to maintain restore compromised functionality (Montesano et al., 2013). The study conducted on a sample of 60 adolescents showed, therefore, the effectiveness of physical activity, related to a correct dietary style, to reduce, even significantly, excess weight with benefits in relational and collaborative behaviors (Comoglio \& Cardoso, 1996). The best results were recorded among male subjects compared to females. The correct physical activity has shown a greater mastery of the respiratory function whose final parameters have improved significantly (Sandip, Murnal, \& Yuganthi, 2012). Intensity training, talent, and an adequate diet represent the crucial factors for the success of athletes and our children (Mazzeo et al., 2016). It is important to educate the adolescent to pursue appropriate lifestyles with the correct use of bodily functions and food hygiene through the exercise of sports activity (Mazzeo et al., 2016; Vengata et al., 2014). A number of strategies (educative and persuasive, facilitative and restrictive) have been proposed to improve the lifestyle to our children. (Mazzeo et al., 2005; Mazzeo et al., 2018). This study is a good starting point for future work in this area of research and in this direction we are going on.
Acknowledgements

There are no acknowledgements.

\section{Conflict of Interest}

The authors declare that there are no conflicts of interest.

Received: 14 October 2018 | Accepted: 23 December 2018 | Published: 01 February 2019

\section{References}

Boulet, L. (2013). Asthma and obesity. Clinical and Experimental Allergy, 43(1), 8-21. doi:10.1111/j.1365-2222.2012.04040

Clinical Guidelines (1998). On the Identification, Evaluation, and Treatment of Overweight and Obesity in Adults-The Evidence Report. National Institutes of Health (NIH). Obes Res, 6(Suppl 2), 51S-209S.

Comoglio M. \& Cardoso, M.A. (1996). Teach and learn in groups. Cooperative Learning (Insegnare e apprendere in gruppo. // Cooperative Learning). LAS, Roma

Farah, C.S., \& Salome, C.M. (2012). Asthma and obesity: A known association but unknown mechanism. Respirology, 17(3), 412-421. doi:10.1111/ j.1440-1843.2011.02080.x

Hill, S.G., Phan, T.T., Datto, G.A., Hossain, J., Werk, L.N., Abatemarco, D. (2018). Integrating childhood obesity resources into the patient-centered medical home: Provider perspectives in the United States. J Child Health Care, 1, 1367493518777308 doi: 10.1177/1367493518777308

Illiano, M., Nigro, E., Sapio, L., Caiafa, I., Spina, A., Scudiero, O.,... \& Naviglio, S. (2017). Adiponectin down-regulates CREB and inhibits proliferation of A549 lung cancer cells. Pulmonary Pharmacology and Therapeutics, 45, 114-120. doi:10.1016/j.pupt.2017.05.009

Luce, J.M. (1980). Respiratory complications of obesity. Chest, 78, 626-30.

Magni, A. (2009). Optimization of coordination skills during intervention times (L'ottimizzazione delle capacità coordinative nei tempi d'intervento), Calzetti e Mariucci, Perugia

Marella, M. \& Risaliti, M. (2007). The Test Book-Evaluation tests for all sports (II libro dei Test - Le prove di valutazioni per tutti gli sport). Ed. Correre, Milano.

Matera, M.G., Rinaldi, B., Calzetta, L., Cazzola, M. (2017) Pharmacogenetic and pharmacogenomic considerations of asthma treatment.ExpertOpinDrug Metab Toxicol, 13(11), 1159-1167. doi: 10.1080/17425255.2017.1391215

Mazzeo, F., Capuano, A., Avolio, A., Filippelli, A., \& Rossi, F. (2005). Hospital-based intensive monitoring of antibiotic-induced adverse events in a university hospital. Pharmacological Research, 51(3), 269-274. doi:10.1016/j.phrs.2004.09.004

Mazzeo, F. (2016). Current concept of obesity. Sport Science, 9(2), 42-48

Mazzeo, F., Santamaria, S., Monda, V., Tafuri, D., Dalia, C., Varriale, L., De Blasio, S., Esposito, V., Messina, G., \& Monda, M. (2016). Dietary supplements use in competitive and non competitive boxer: An exploratory study. Biology and Medicine, 8(4), 294.

Mazzeo, F., Monda, V., Santamaria, S., Nigro, E., Valenzano, A., Villano, I.,... \& Messina, G. (2018). Antidoping program: An important factor in the promotion and protection of the integrity of sport and athlete's health. Journal of Sports Medicine and Physical Fitness, 58(7-8), 1135-1145. doi:10.23736/S0022-4707.17.07722-2

Montesano, P. (2016). Goalkeeper in soccer: performance and explosive strength. Journal of Physical Education and Sport, 16(1), 230 - 233. doi:10.7752/jpes.2016.01036

Montesano, P., Tafuri, D., Esposito, A., Gigante, F., Salzano, E., Viscido, G., \& Mazzeo, F. (2013). Conditional abilities in young special olympics athletes who practice unified football. Journal of Physical Education and Sport, 13(4), 504-510. doi:10.7752/jpes.2013.04079

Montesano, P. \& Mazzeo, F. (2018). Pilates Improvement the Individual Basics of Service and Smash in Volleyball. Sport Mont, 16(3), 25-30. doi: 10.26773/smj.181005

Montesano, P., (2018). Monitoring and upgrading of coordinative capacities of young athletes practicing handball. Journal of Physical Education and Sport, 18(1), 465-468. doi:10.7752/jpes.2018.s165

Oates, G.R., Phillips, J.M., Bateman, L.B., Baskin, M.L., Fouad, M.N., \& Scarinci, I.C. (2018). Determinants of obesity in two urban CommUnities: PerCePtions anD CommUnity-driven solutions. Ethnicity and Disease, 28(1), 3342. doi:10.18865/ed.28.1.33

Parameswaran, K., Todd, D.C., \& Soth, M. (2006). Altered respiratory physiology in obesity. Journal of the Canadian Thoracic Society, 13(4), 203-210. doi:10.1155/2006/834786

Perrotta, F., Mazzeo, F., \& Cerqua, F.S. (2017). Which treatment for obstructive airway disease: The inhaled bronchodilators. Pulmonary. Pharmacology and Therapeutics, 43, 57-59. doi:10.1016/j.pupt.2017.01.003

Persson, P.B., Persson, A.B (2018). Metabolism, obesity and the metabolic syndrome. Acta Physiol (Oxf), 223(3), e13096. doi: 10.1111/apha.13096. 
Ruiz, J.R., Ortega, F.B., Castillo, R., Martín-Matillas, M., Kwak, L., Vicente-Rodrí guez, G.,... Moreno, L.A. (2010). Physical activity, fitness, weight status, and cognitive performance in adolescents. Journal of Pediatrics, 157(6), 917-922.e5. doi:10.1016/j.jpeds.2010.06.026

Salome, C.M., King, G.G., Berend, N. (2010). Physiology of obesity and effects on lung function. J Appl Physiol, 108, 206-211. doi: 10.1152/japplphysiol.00694.2009.

Sandip, M., Murnal, S., Yuganthi, P. (2012). Cardiorespiratory response to aerobic exercise programs with different intensity: 20 weeks longitudinal study. J Res Med Sci., 17, 649-655.

Schenone, S., Bruno, O., Ranise, A., Brullo, C., Bondavalli, F., Filippelli, W.,... Falcone, G. (2003). 2-aryl-3-phenylamino-4,5-dihydro-2h-benz[g]indazoles with analgesic activity. Farmaco, 58(9), 845-849. doi:10.1016/S0014827X(03)00142-3

Vengata Subramanai, M., Ganesh Sundaram, S., \& Wan Rosley, W. (2014). Effect of physical activity on respiratory function in obese: a systematic review. Int J Physiother Res, 2(1), 342-46.
Vogler, E.W., Koranda, P., \& Romance, T. (2000). Including a child with severe cerebral palsy in physical education: A case study. Adapted Physical Activity Quarterly, 9, 316-329. doi: 10.1123/apaq.17.2.161

Wells, G.D., Noseworthy, M.D., Hamilton, J., Tarnopolski, M., \& Tein, I. (2008). Skeletal muscle metabolic dysfunction in obesity and metabolic syndrome Can J Neurol Sci., 35(1), 31-40.

WHO. (2013). Obesity and overweight. Retrieved from https://www.who.int/ gho/publications/world_health_statistics/2013/en/

Yazdani, N., Sharif, F., Elahi, N., Ebadi, A., \& Hosseini, S.V. (2018). Psychometric properties of quality of life assessment tools in morbid obesity: A review of literature. Evidence Based Care, 7(4), 7-21. doi:10.22038/ebcj.2017.26221.1606

Zametkin, A.J., Zoon, C.K., Klein, H.W., \& Munson, S. (2004), Psychiatric aspects of child and adolescent obesity: a review of the past 10 years, J Am Academy of Child \& Adolescent Psychiatry, 43(2), 134-50. doi:10.1097/00004583-200402000-00008 